“NOTICE: this is the author's version of a work that was accepted for publication in International Journal of Impact Engineering. Changes resulting from the publishing process, such as peer review, editing, corrections, structural formatting, and other quality control mechanisms may not be reflected in this document. Changes may have been made to this work since it was submitted for publication. A definitive version was subsequently published in INTERNATIONAL JOURNAL OF IMPACT ENGINEERING, VOL 38, ISSUE 10, OCTOBER 2011, PAGES 815-823, DOI: 10.1016/j.ijimpeng.2011.05.005”

\title{
Hard projectile penetration and trajectory stability
}

\author{
Q.M. Li ${ }^{1, *}$, E.A. Flores-Johnson ${ }^{2}$ \\ ${ }^{1}$ School of Mechanical, Aerospace and Civil Engineering, Pariser Building, The University of Manchester \\ Sackville Street, Manchester M13 9PL, UK \\ ${ }^{2}$ Institute of Materials Engineering, Australian Nuclear Science and Technology Organisation \\ Locked Bag 2001, Kirrawee DC, NSW, 2232 Australia
}

\begin{abstract}
We present a general framework to describe the dynamics of a hard projectile penetrating into a solid target. Rigid-body dynamics, differential area force law and semi-empirical resistance function are used to formulate the motion of the hard projectile. The proposed model is capable of predicting the projectile trajectory under various oblique and yaw angles. Critical conditions for the occurrences of the instability and the reverse of the projectile trajectory are discussed. It was found that the relative location of mass centre of the projectile has strong influence on the control of the rotation of the projectile, and thus, the projectile stability and the change of trajectory direction. The validity of the proposed model is limited to deep penetration and when the wake separation and reattachment between projectile body and target have negligible influence on the target resistance to the projectile.
\end{abstract}

Keywords: penetration, rigid projectile, trajectory stability, soil target 


\section{Introduction}

When deformation and failure of a kinetic projectile is negligible in a penetration process, the motion of the projectile and its penetration capability can be analysed by considering the projectile as a hard projectile based on the rigid-body assumption. Extensive researches have been done on the penetration of hard projectiles into various targets [e.g. see further details in Forrestal and Luk(1992) for soil target, Chen and $\mathrm{Li}(2002)$ for metallic target, Li et al.(2005) for concrete target, Li et al.(2005) and Ahmad Zaidi and Li(2009) for cellular target].

An analytical method, termed differential area force law (DAFL), was proposed by the AVCO Corporation in early 1970s to provide explicit formulations for the normal and tangential stresses on the projectile surface [Heuze(1990)]. The DAFL approach was adopted and modified by the US Army Waterways Experiment Station (WES) to provide 2-D (PENCO2D) and 3-D (PENCRV3D) codes for projectile trajectory analyses [Adley et al.(1997), Danielson and Adley(2000)].

A general framework of hard projectile penetration was presented in $\mathrm{Li}(2004)$ based on DAFL approach. This framework is capable of studying deep penetration problems when non-deformable projectile penetration regime is maintained [Chen and Li(2004)]. Particularly, it can be used to study the curvilinear motion of the projectile and its stability during deep penetration.

Projectile trajectory during deep penetration does not always follow a straight path. Under certain circumstances, the projectile may have a curved or J-shape trajectory that may or may not result in a reverse of trajectory direction (i.e., projectile moves toward the target surface). Curved trajectories are more likely to occur in oblique impact and/or yawed impact when rotational velocity is introduced to the projectile due to the initial non-axisymmetric surface contact when the projectile starts to interact with the target medium. However, in normal impact when certain lateral disturbances are introduced to the projectile during flight such as a small oblique angle or yaw angle or a small rotational velocity, the trajectory may start to deviate from the normal direction under certain conditions, which can be considered as an instability problem.

Projectile trajectory and stability are important issues in the prediction of the terminal location of a kinetic projectile. There are limited investigations on these issues [Bernard and Creighton(1979), Simonov and Osipenko(2004)], and many key questions remain inconclusive. A general 3-D model for rigid projectile dynamics will be presented and validated in this paper, which will then be used to analyse the trajectory and stability of the projectile. In Section 2, we will briefly describe the framework of hard projectile penetration in $\mathrm{Li}(2004)$ with further discussion because this resource cannot be easily accessed. This framework is validated in Section 3 before it is used to study the curvilinear motion of the projectile and its stability in soil target in Section 4. Finally, Section 5 provides conclusive remarks.

\section{Framework of hard projectile penetration}

The hard projectile in consideration is simplified into a rigid body, which has a revolutionary body with an outward-concave surface. The theoretical framework can be extended to irregular rigid projectiles when introducing more advanced contact conditions. The limitation of the rigid projectile assumption will not be discussed. Interested readers may refer to other publications [e.g. Chen and Li (2004)] to judge the validity of this assumption.

The orientation and position of the revolutionary rigid body at time $t$ is shown in Fig.1. The fixed reference frame is represented by $[\vec{x}, \vec{y}, \vec{z}]$ and the rigid body reference frame located at its 
centroid is represented by $\left[\vec{e}_{1}^{\prime}, \vec{e}_{2}^{\prime}, \vec{e}_{3}^{\prime}\right]$. An intermediate reference frame, $\left[\vec{e}_{1}, \vec{e}_{2}, \vec{e}_{3}\right]$, is introduced, which has the same origin as the rigid body reference frame, but does not rotate. $C$ is the centroid of the rigid body and $I_{1}, I_{2}$ and $I_{3}$ are the three principal moments of inertia about axes of $\left[\vec{e}_{1}^{\prime}, \vec{e}_{2}^{\prime}, \vec{e}_{3}^{\prime}\right]$. It is obvious that $I_{2}=I_{3}$ for a revolutionary projectile.

The motion of the hard projectile is controlled by the rigid body dynamics, i.e.

$$
\begin{aligned}
& M \frac{d \vec{V}_{c}}{d t}=\vec{F}_{R}, \\
& \left(\frac{d \vec{L}_{c}}{d t}\right)_{\vec{e}^{\prime}}+\vec{\omega} \times \vec{L}_{c}=\vec{N}_{c},
\end{aligned}
$$

together with the initial conditions of the translation and angular velocities and the positions of the projectile. In Eqs.(2) and (3), $M$ is the total mass of the projectile, $\vec{V}_{c}$ is the velocity of the centroid of the projectile, $\vec{\omega}=\omega_{1} \vec{e}_{1}^{\prime}+\omega_{2} \vec{e}_{2}^{\prime}+\omega_{3} \vec{e}_{3}^{\prime}$ is the angular velocity of the projectile, $\left(\frac{d}{d t}\right)_{\vec{e}^{\prime}}$ represents the time derivative with respect to the rigid body reference frame, $\vec{L}_{c}$ is the angular momentum about the centroid of the projectile, determined by

$$
\vec{L}_{c}=I_{1} \omega_{1} \vec{e}_{1}^{\prime}+I_{2} \omega_{2} \vec{e}_{2}^{\prime}+I_{3} \omega_{3} \vec{e}_{3}^{\prime} .
$$

$\vec{F}_{R}$ and $\vec{N}$ are the resultant resistant force and the resultant moment of the contact forces about the centroid of projectile, respectively, which are applied to the surface of the projectile by surrounding target media and depend on the contact resistance of the target medium.

Generally, the contact resistance of the target media consists of normal resistance and tangential resistance. If the unit vector of the normal direction of the projectile surface at point $P$ is $\vec{n}$, as shown in Fig.2, it can be determined by

$$
\vec{n}=\left(\frac{\partial f}{\partial x_{1}} \vec{e}_{1}^{\prime}+\frac{\partial f}{\partial x_{2}} \vec{e}_{2}^{\prime}+\frac{\partial f}{\partial x_{3}} \vec{e}_{3}^{\prime}\right)_{P}
$$

where $f\left(x_{1}, x_{2}, x_{3}\right)$ is the function to represent the out-surface of the projectile in the rigid body reference frame $\left[\vec{e}_{1}^{\prime}, \vec{e}_{2}^{\prime}, \vec{e}_{3}^{\prime}\right]$. The stress vector of the contact resistance at point $P$ is determined by two components, i.e. the normal stress and tangential stress, given by

$$
\vec{\sigma}_{n}=-H\left(V_{n}\right) \sigma_{n} \vec{n} \quad \text { and } \quad \vec{\tau}_{n}=\mu\left(V_{\tau}\right) H\left(V_{n}\right) \sigma_{n} \vec{\tau}
$$

where $\sigma_{n}$ is the normal stress at point $P$ on the out-surface of the projectile, which will be discussed later. The Heaviside function $H\left(V_{n}\right)$ represents the unilateral condition of the contact, i.e.,

$$
H\left(V_{n}\right)=0 \quad \text { if } \quad V_{n}<0 \text { and } H\left(V_{n}\right)=1 \quad \text { if } \quad V_{n} \geq 0 .
$$

$V_{n}$ and $V_{\tau}$ are the normal and tangential components of the particle velocity of the point $P$ on the surface of the projectile, given by

$V_{n}=\vec{V} \cdot \vec{n}$ and $V_{\tau}=\vec{V} \cdot \vec{\tau}$

in which the unit vector of the tangential direction $\vec{\tau}$ is determined by (see Fig.2) 


$$
\vec{\tau}=\vec{m} \times \vec{n} \text { and } \vec{m}=\vec{n} \times\left(\frac{\vec{V}}{\|\vec{V}\|}\right)
$$

The above formulation assumes that the contact resistance can be divided into normal resistance that depends on the normal velocity, and the friction resistance that depends on the relative tangential velocity. $\mu$ is the kinetic friction coefficient, which is a function of the relative sliding velocity (i.e. the tangential velocity $V_{\tau}$ ) between the target and the projectile. Therefore, the resultant resistant force and moment in Eqs.(2) and (3) are

$$
\vec{F}_{R}=\iint_{\Omega}\left(\vec{\sigma}_{n}+\vec{\tau}_{n}\right) d S \text { and } \vec{N}_{c}=\oiint_{\Omega} \vec{r}_{c p} \times\left(\vec{\sigma}_{n}+\vec{\tau}_{n}\right) d S
$$

where $\Omega$ is the domain of the projectile outer surface and $\vec{r}_{c p}$ is the position vector from point $C$ to point $P$. When the target consists of multiple media, the target space is represented by $V=\bigcup_{j i ̂} 1, N$ where $V_{j}$ represents $j$ - th medium with specified geometrical domain and mechanical properties. In this paper, we will focus on single medium target. However, the methodology can be easily extended to a target consisting of multiple media.

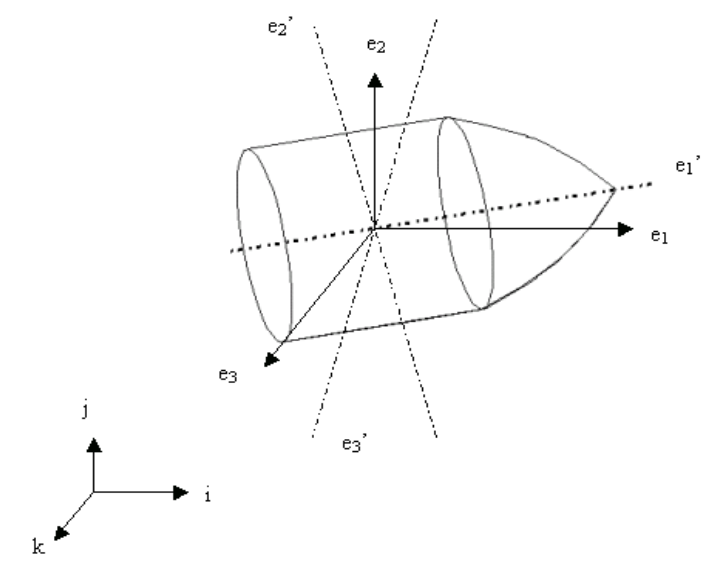

Fig.1 Reference frames for the projectile motion

Various resistant functions have been suggested for different target materials. It is well known that the gas and fluid resistance depends on the relative velocity between the projectile and the gas or fluid medium. For a solid target, a strength term [i.e. parameter $a_{0}$ in Eq.(11)] has to be added. Thus, the commonly used resistant function for solid target can be expressed as a polynomial function of normal velocity [Li et al.(2005)], i.e.

$$
\sigma_{n}=a_{0}+a_{1} V_{n}+a_{2} V_{n}^{2}+\ldots \ldots
$$

Within the above-introduced framework, the trajectory of the projectile, penetration depth, trajectory stability and the optimal design of the projectile nose shape can be analysed. 


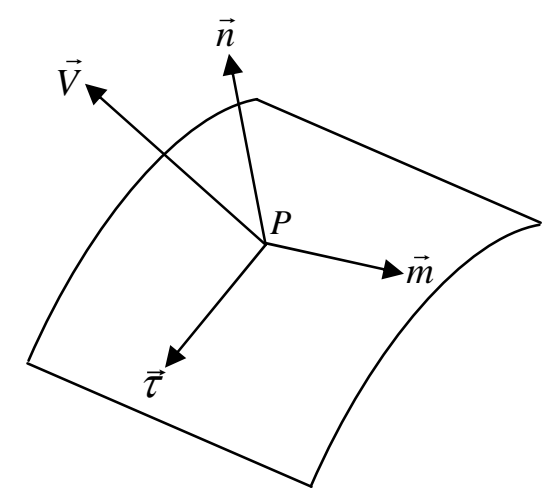

Fig.2 Orientation of the projectile surface

It should be noted that although the free surface is considered in this investigation by defining the interface between different target media, free-surface effects on the penetration resistance near the surface of the target are not considered in this framework. Penetration resistance formulae used for deep penetration and near surface penetration are different, as reported in many publications [e.g. Bernard and Creighton(1979), Bless et al. (1999), Warren et al. (2004), Li and Chen(2003),]. Freesurface effects are important under certain conditions, e.g., brittle target medium (concrete or rock), long-rod projectile penetration [Bless et al. (1999)], large impact oblique angles [Warren et al. (2004)], and shallow penetration depth [Liu et al. (2009)]; however, for the target medium used in this investigation (soil), free-surface effects are less significant since soils are more ductile than brittle in behaviour [Bernard and Creighton (1979)]. Because this study will focus on deep penetration problems, it is legitimate to ignore the free surface effects. However, when the penetration resistance is specified near the surface of the target, it can be easily implemented into the framework.

Depending on the projectile nose shape, impact velocity and the mechanical properties of the target, target may be detached from the surface of the projectile body (normally the shank or aftbody of the projectile) during penetration, which is termed as wake separation [Bernard and Creighton(1978)]. Erosion patterns on recovered projectiles also indicate that the reattachment of the target medium to the projectile surface may occur [Byers et al.(1978)]. Although simplified model has been proposed to consider these issues in penetration analysis, the comparison between calculated and experimental results is inconclusive [Bernard and Creighton(1979)]. Therefore, wake separation and reattachment will not be included in the present study. However, they can be easily added to the framework in this section when better understanding on the physics of wake separation and reattachment is obtained.

\section{Implementation and validation of the framework}

The framework of hard projectile penetration introduced in Section 2 was implemented in ABAQUS explicit via the user subroutine VDLOAD, which is used to define a distributed pressure $\sigma_{n}$ on the outer surface of the projectile described as a discrete rigid body (Fig.3). VDLOAD is called in each time step and calculates the pressure to be applied on each surface element or at the integration point $i$ where the distributed load is defined. Figure 4(a) shows a schematic diagram of this process in which ABAQUS explicit calls VDLOAD. Figure 4(b) shows a flowchart of the algorithm coded in Fortran to implement the framework via VDLOAD. As the first step in the 
algorithm, if the global $y$-coordinate of a surface element $i$ is greater than zero, the load is equal to zero which means that that particular projectile element has not reached the surface of the meshless target domain defined at $y=0$ (Fig.3). If the global $y$-coordinate is less or equal to zero, then the unilateral contact condition defined in Eq.(7) is evaluated. If $V_{n} \geq 0$, the resistant function defined in Eq.(11) is applied to the surface element. The process is repeated until all surface elements of the projectile are processed by the user subroutine. The reader is referred to the ABAQUS Analysis User's Manual [ABAQUS(2004)] for further information of VDLOAD user subroutine.

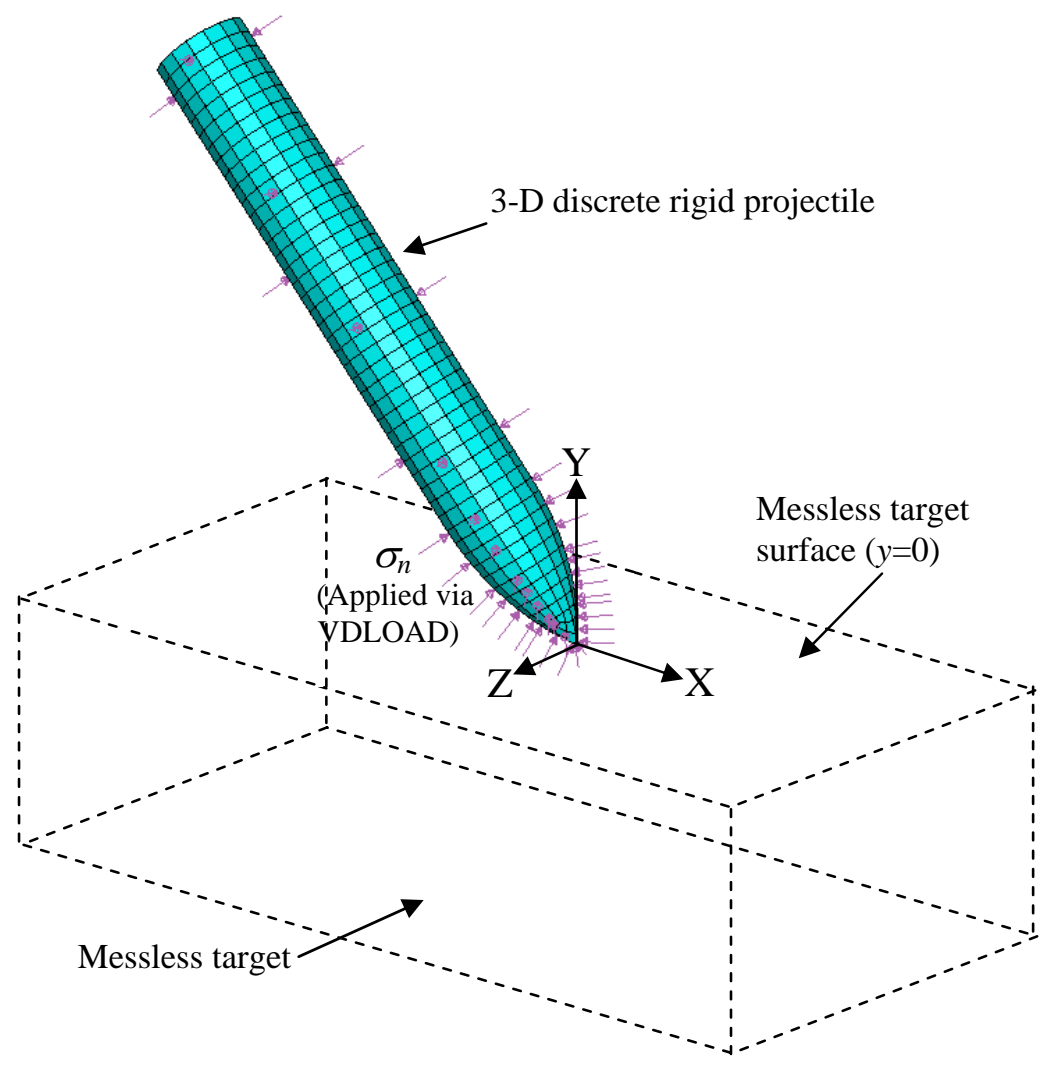

Fig.3 Finite element model

To validate the implementation of the framework, comparisons between selected experimental data of various targets under normal and oblique impact and numerical simulations are made. Table 1 summarizes the properties and the implemented resistant functions for various targets, i.e., concrete, aluminium-alloy and soil. Figure 5 shows the comparisons for concrete and aluminiumalloy targets. It can be seen that the predictions of penetration depth into concrete targets by a projectile with calibre radius head (CRH) of 4.25 agree very well with experimental results for all impact velocities. When $\mathrm{CRH}=3$, the penetration depth is overestimated up to $7.5 \%$ for the highest impact velocity. It can be seen in Fig. 5 that the relative difference increases with the increase of the impact velocity. This overestimation may be attributed to the assumptions that there is no friction between the projectile and the target and that the projectile behaves as a rigid body because both factors reduce the penetration depth. For aluminium-alloy targets, the penetration depth is well predicted for velocities below $600 \mathrm{~m} / \mathrm{s}$. For higher velocities, predictions overestimate the penetration depth with a relative difference that increases with the increase of impact velocity. It is noticeable that the relative difference for $\mathrm{CRH}=0.5$ and velocities greater than $1000 \mathrm{~m} / \mathrm{s}$ is very large. This may be attributed to the fact that there exists a transition of the projectile behaviour from 
rigid body behaviour to semi-hydrodynamic behaviour in which the projectile erosion and/or deformation leads to a reduction in the penetration depth. This transition has been observed experimentally for steel projectiles and aluminium-alloy targets [Forrestal and Piekutowski(2000)] and has been analysed in Chen and Li (2004).

Table 1 Target parameters and resistance functions

\begin{tabular}{lll}
\hline Target & Resistance function & Parameters \\
\hline Concrete [Forrestal et al.(1994)] & $\sigma_{n}=\mathrm{S} f_{\mathrm{c}}+\rho V_{n}^{2}$ & $\mathrm{~S}=21, f_{\mathrm{c}}=13.5 \mathrm{MPa}, \rho=1960 \mathrm{~kg} / \mathrm{m}^{3}$ \\
$\begin{array}{l}\text { Aluminium [Piekutowski et al.(1999), } \\
\text { Forrestal and Piekutowski(2000)] }\end{array}$ & $\sigma_{n}=\mathrm{AY}+\mathrm{B} \rho V_{n}^{2}$ & $\mathrm{~A}=5.04, \mathrm{~B}=0.983, \mathrm{Y}=276 \mathrm{MPa}, \rho=2710 \mathrm{~kg} / \mathrm{m}^{3}$ \\
Soil [Forrestal and Luk(1992)] & $\sigma_{n}=\mathrm{A} \tau_{0}+\mathrm{B} \rho V_{n}^{2}$ & $\mathrm{~A}=1.718, \mathrm{~B}=2.215, \tau_{0}=10 \mathrm{MPa}, \rho=1860 \mathrm{~kg} / \mathrm{m}^{3}$ \\
\hline
\end{tabular}
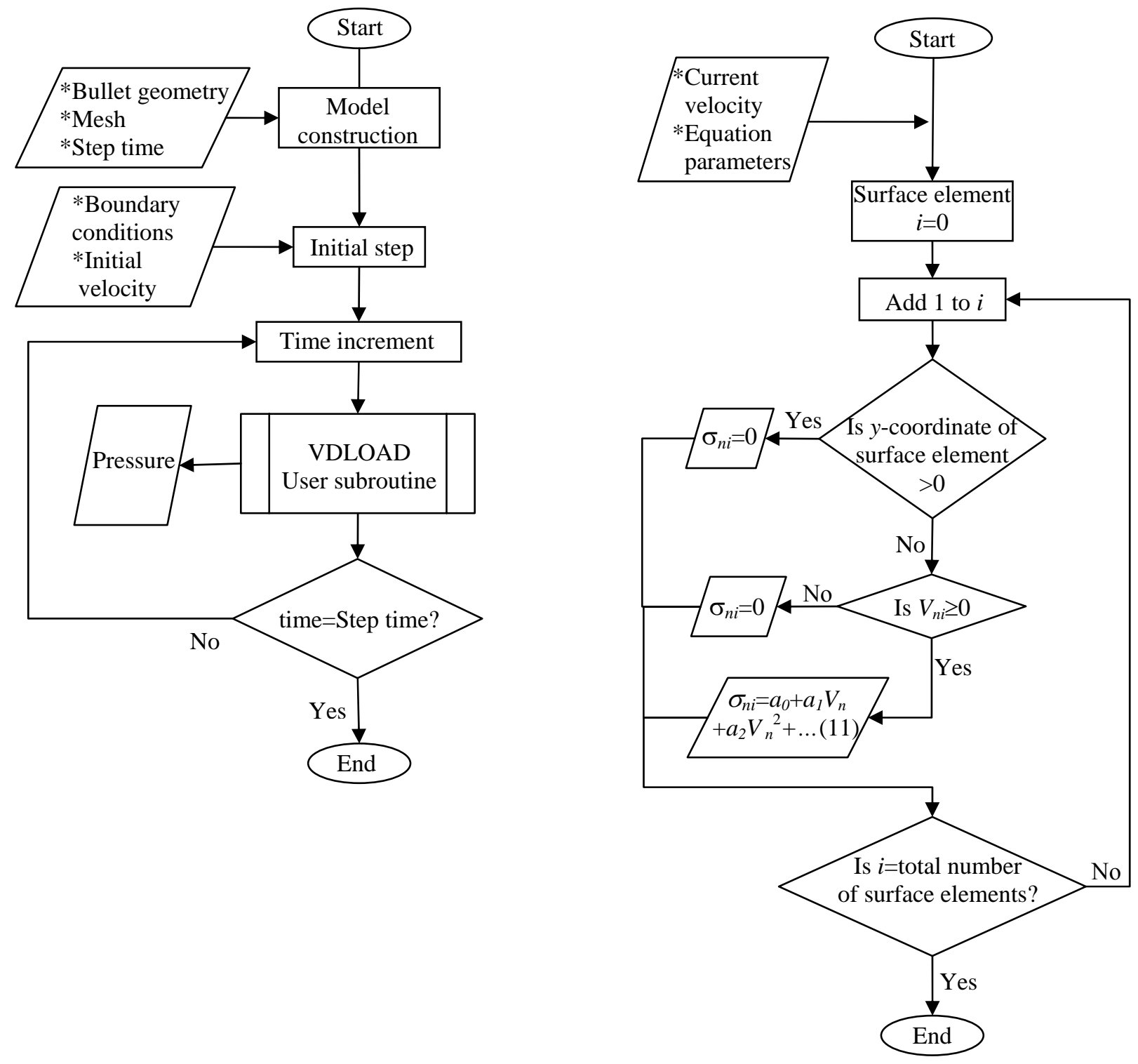

Fig.4 Flowchart of a) Process of ABAQUS explicit calling VDLOAD user subroutine, b) Framework implementation using VDLOAD 
Comparison between experimental and numerical results of penetration depth for soil targets are shown in Table 2. The maximum relative difference between experimental and predicted penetration depth is $18.7 \%$. This difference may be attributed to experimental uncertainty or due to the change of soil properties with depth, which were not considered [Forrestal and Luk(1992)].

In general, the present method gives reasonably good predictions for a range of target media and impact velocities when the assumptions of the theoretical framework can be satisfied.

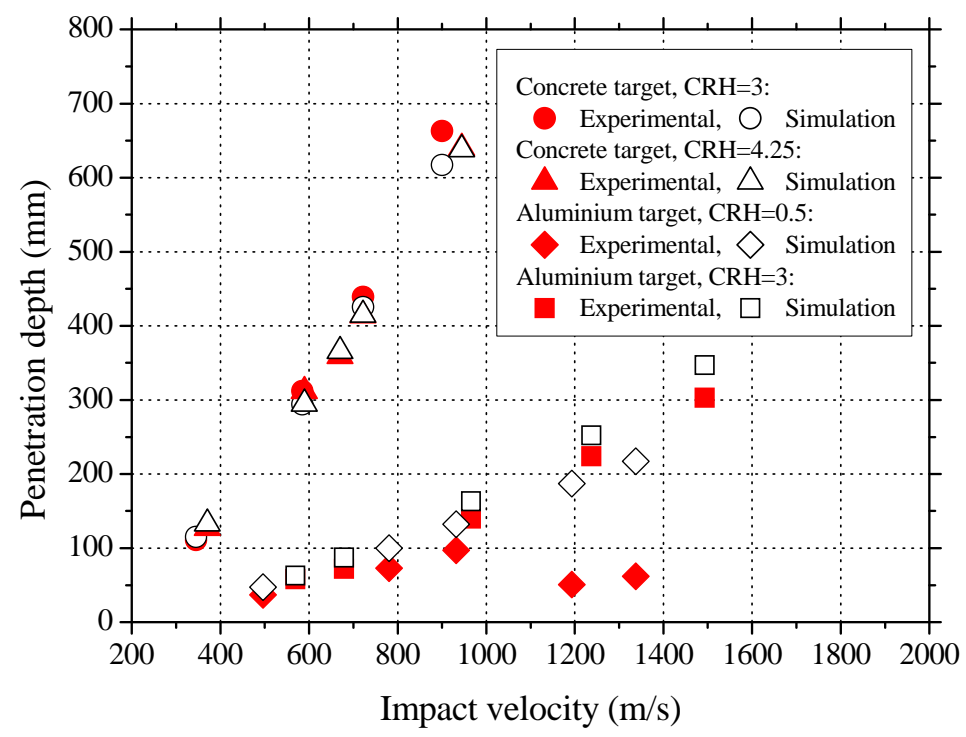

Fig.5 Comparison between experimental results for concrete targets [Forrestal et al.(1994)] and aluminium-alloy targets [Piekutowski et al.(1999), Forrestal and Piekutowski(2000)] and numerical simulations

Table 2 Comparison between experimental [Forrestal and Luk(1992)] and predicted penetration depths for soil target.

\begin{tabular}{cccccc}
\hline Test \# & $\begin{array}{c}\text { Impact velocity } \\
(\mathrm{m} / \mathrm{s})\end{array}$ & $\begin{array}{c}\text { Angle of obliquity } \\
\left({ }^{\circ}\right)\end{array}$ & $\begin{array}{c}\text { Yaw angle } \\
\left({ }^{\circ}\right)\end{array}$ & $\begin{array}{c}\text { Experimental penetration } \\
\text { depth (m) }\end{array}$ & $\begin{array}{c}\text { Predicted penetration } \\
\text { depth }(\mathrm{m})\end{array}$ \\
\hline 2 & 280 & 0 & 0 & 4.98 & 4.25 \\
3 & 278 & 30 & 0 & 5.18 & 4.21 \\
4 & 280 & 30 & 0 & 5.18 & 4.25 \\
5 & 280 & 0 & 3.5 & 5.02 & 4.22 \\
6 & 280 & 0 & 4.0 & 4.82 & 4.21 \\
\hline
\end{tabular}

\section{Instability and curvilinear motion of projectile trajectory}

\subsection{Introduction}

We first clarify several concepts in the study of the trajectory of a hard projectile in target medium. When a revolutionary hard projectile hits a homogenous target at normal angle with little lateral disturbances (e.g. yaw, pitch etc.) or travels in one dimensional (1-D) linear motion in a homogenous target, the resultant force applied on the outer surface of the projectile is in the opposite direction of its linear velocity. Instability occurs if the projectile motion deviates from the 1-D linear motion to a curvilinear motion. The conditions, under which the instability occurs, are called instability conditions. For example, if all other conditions are fixed, projectile instability may occur at a critical impact velocity $\left(V_{c i}\right)$, which is termed as the critical velocity for the occurrence of projectile instability. Practically, lateral disturbances and imperfections are unavoidable to destroy the axisymmetric nature of the penetration problem. These lateral disturbances and imperfections 
may include yaw, pitch and oblique angles, rotating velocities, geometric imperfections, etc., but only if their magnitudes are several orders smaller than corresponding axisymmetric quantities. Otherwise, the penetration should be considered as a non-axisymmetric penetration problem and the concept of instability is not applicable. This is a common methodology when dealing with dynamic instability problems [Lindberg HE and Florence AL(1987)].

For non-axisymmetric penetration problems (e.g. with relatively large oblique, yaw and/or pitch angles, or with non-revolutionary projectile body), curvilinear motion is expected from the very beginning. For this type of problem, a practical important scenario is that the projectile motion reverses toward the target surface. The conditions, under which the reverse motion of a projectile occurs, are called critical reverse conditions. For example, if all other conditions are fixed, projectile reverse may occur at a critical oblique angle. Experimental [Rosenberg et al.(2005)] and numerical [Dorogoy et al.(2010)] investigations on oblique impact of polymethylmetracrylate plates, show that projectile reverse occurs for large angles of obliquity $\left(\geq 60^{\circ}\right)$ when the plates are impacted by a 0.3 ” AP projectile with an impact velocity of $720 \mathrm{~m} / \mathrm{s}$.

Deep penetration of projectiles into soil targets was reported experimentally and numerically by Bernard and Creighton(1979). They reported that the trajectory of the projectile went from linear to curvilinear when the impact velocity was increased from 427 to $512 \mathrm{~m} / \mathrm{s}$ for small angles of obliquity $\left(3.7^{\circ}\right)$. Bernard and Creighton(1979) also reported that for larger angles of obliquity $\left(>30^{\circ}\right)$, the projectile trajectory was curvilinear and may move toward the target surface when the projectile slenderness ratio $L / D$ (where $L$ and $D$ are the length and diameter of the projectile, respectively) was reduced, which implied that the projectile trajectory can be largely influenced by its geometry. The former belongs to a stability problem, and the latter belongs to projectile reverse in a curvilinear motion.

Simonov and Osipenko(2004) reported an analytical study on the trajectory instability of hard blunt projectiles in low density rock medium for normal impact. They found that projectile instabilities can occur for large yaw angles. They demonstrated the reverse of the projectile motion and found that the curvature of the curvilinear trajectory of the projectile increased with the distance of the projectile mass centre from its nose tip. Bernard and Creighton(1979) found that short projectile $(L / D=8)$ is less stable than longer projectiles $(L / D=10)$ when they analysed soil penetration data for non-normal impact conducted at Sandia National Laboratories [see Table 3.1 in Bernard and Creighton(1979)]. A detailed examination of experimental data shows that the former case corresponds to $L_{c} / L=0.58$ while latter cases correspond to $L_{c} / L=0.50-0.53$ (where $L_{c}$ is the distance from the tip of the nose to the centre of mass). We believe that $L_{C} / L$ is a dominant parameter to control the stability of the projectile, which will be further investigated in this paper.

In this investigation, the mechanical properties and penetration resistance for soil targets described in Table 1 are used to investigate the trajectory instability and curvilinear trajectory in deep penetration. The resistance function was implemented in ABAQUS as described in Section 3. The resistance function is based on a simplification of the cavity-expansion theory for a MohrCoulomb Tresca-limit material [Forrestal and Luk(1992)] with the assumption of non-friction between the projectile and the target. Material properties in Table 1 for soil target are average values, which do not represent the variation of soil properties with depth. This introduces uncertainty in the material properties. However, the comparison between numerical simulations and experimental results in Table 2 shows a discrepancy of less than 20\% which is acceptable for the given experimental/material properties uncertainties. 


\subsection{Curved trajectory and critical reverse velocity}

The effect of the angle of obliquity in the projectile trajectory of deep penetration into soil target is investigated for a range of impact velocities. The geometry of the projectile used is shown in Fig.6. To study the effect of the location of the centre of mass on the motion of the projectile, different densities were assigned to the shank of the projectile (Table 3 ) to change the values of $L_{c} / L$ between 0.504 and 0.571 . The density of the projectile nose was fixed in all simulations as 7850 $\mathrm{kg} / \mathrm{m}^{3}$.

Figure 7(a) shows the trajectories for different impact velocities using projectile \#2 and an angle of obliquity $\phi_{1}=45^{\circ}$. The tip of the projectile nose is located at $(x=-1, y=1)$ at the beginning of the simulation. To analyze the trajectories of Fig.7(a), we employed the first derivative $d x / d y$ of the trajectories, as shown in Fig.7(b). It can be seen that all trajectories are linear at the beginning of the simulation before the projectile enters into the soil target with $\mathrm{d} x / \mathrm{d} y=1$ which corresponds to $\tan \left(45^{\circ}\right)$. A change in the trajectory direction occurs when the projectile enters the target medium. The duration of the change of trajectory direction increases with the increase of the impact velocity when other parameters are fixed. Eventually, the curvilinear motion turns into linear motion when $\mathrm{d} x / \mathrm{d} y$ becomes a constant. It suggests that for large angles of obliquity the change of trajectory direction is initiated by the initial non-axisymmetric loading distribution rather than instability. For $V_{i}=1641,1650$ and $1700 \mathrm{~m} / \mathrm{s}$, the projectile trajectory changes from curvilinear motion to linear motion at $x$-displacement of approximate $7 \mathrm{~m}$. It is also observed that for $V_{i}=1650$ and $1700 \mathrm{~m} / \mathrm{s}$ the projectile moves back to the surface $(\mathrm{d} x / \mathrm{d} y>0)$. A critical reverse velocity $V_{c r}$ is defined as the velocity at which the projectile starts to turn back to the surface when $\mathrm{d} x / \mathrm{d} y=0$.

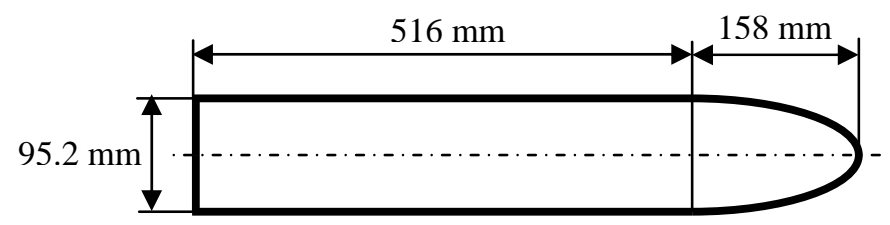

Fig. 6 Ogive-nose projectile geometry $(\mathrm{CRH}=3)$
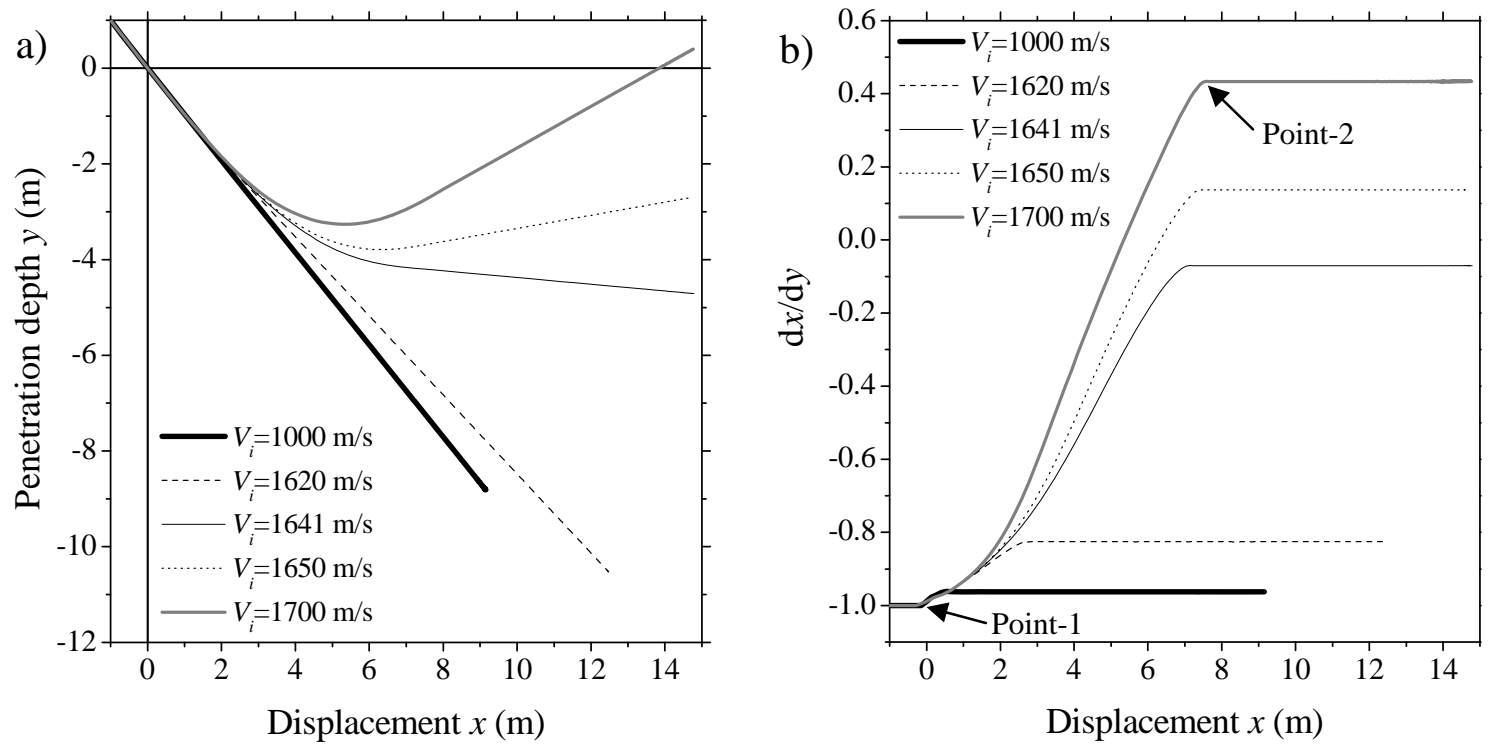

Fig.7 (a) Trajectories for projectile $\# 2$ and $\phi_{1}=45^{\circ}$, b) $d x / d y$ of the trajectories from Fig.7(a) 
Table 3 Description of projectiles with geometry described in Fig.6

\begin{tabular}{cccc}
\hline Projectile \# & Mass $(\mathrm{kg})$ & Density of shank $\left(\mathrm{kg} / \mathrm{m}^{3}\right)$ & $L_{\mathrm{c}} / L$ \\
\hline 1 & 19.1 & 4000 & 0.504 \\
2 & 23.1 & 5150 & 0.525 \\
3 & 32.8 & 7850 & 0.553 \\
4 & 45.3 & 11340 & 0.571
\end{tabular}

Note: $L_{\mathrm{c}}=$ distance from the tip of the nose to the centre of mass, $L=$ total length of the projectile.

Figure 8 shows the dependency of $V_{c r}$ on $\phi_{1}$ for various projectiles. It can be seen that $V_{c r}$ decreases when $\phi_{1}$ is increased. It can also be observed that $V_{c r}$ decreases when $L_{c} / L$ is increased for a given $\phi_{1}$. This shows that the trajectory is more likely to change direction when the centre of mass is further from the tip of the projectile nose.

To qualitatively describe the influence of $L_{c} / L$ on the curvilinear motion of a projectile, we define a total direction change $\Delta \theta_{12}$ (or total turning angle) between Point- 1 and Point- 2 on the projectile trajectory by

$$
\Delta \theta_{12}=\theta_{2}-\theta_{1}
$$

where $\theta_{1}$ and $\theta_{2}$ are the angles between the normal direction and the axis of symmetry of the projectile at Point- 1 and Point-2, respectively. Values of $\Delta \theta_{12}$ for the four projectiles in Table 3 with oblique angle of $45^{\circ}$ and impact velocity of $1000 \mathrm{~m} / \mathrm{s}$ are calculated and presented in Fig.9. Points-1 and -2 are determined by the $\mathrm{d}^{2} x / \mathrm{d} y^{2}=0$ as indicated in Fig.7(b). Figure 9 shows that $\Delta \theta_{12}$ increases with the increase of $L_{c} / L$. Since $\Delta \theta_{12}$ represents the total turning angle of the projectile, it implies that the rotation of a projectile with a larger value of $L_{c} / L$ becomes easier.

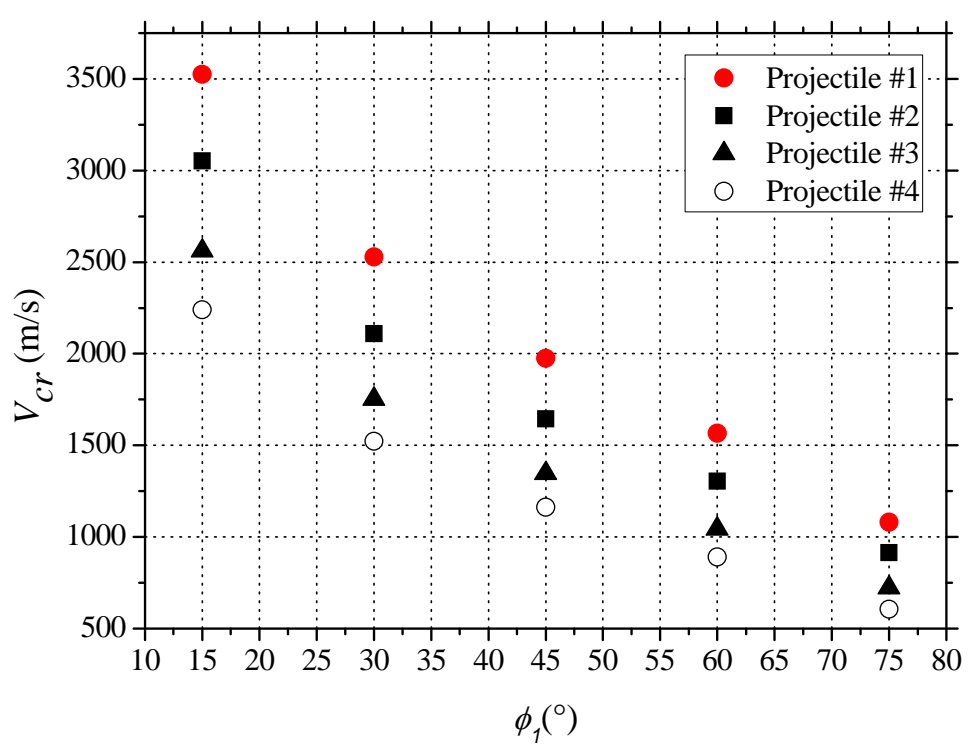

Fig.8 Critical reverse velocity vs angle of obliquity for various projectiles 


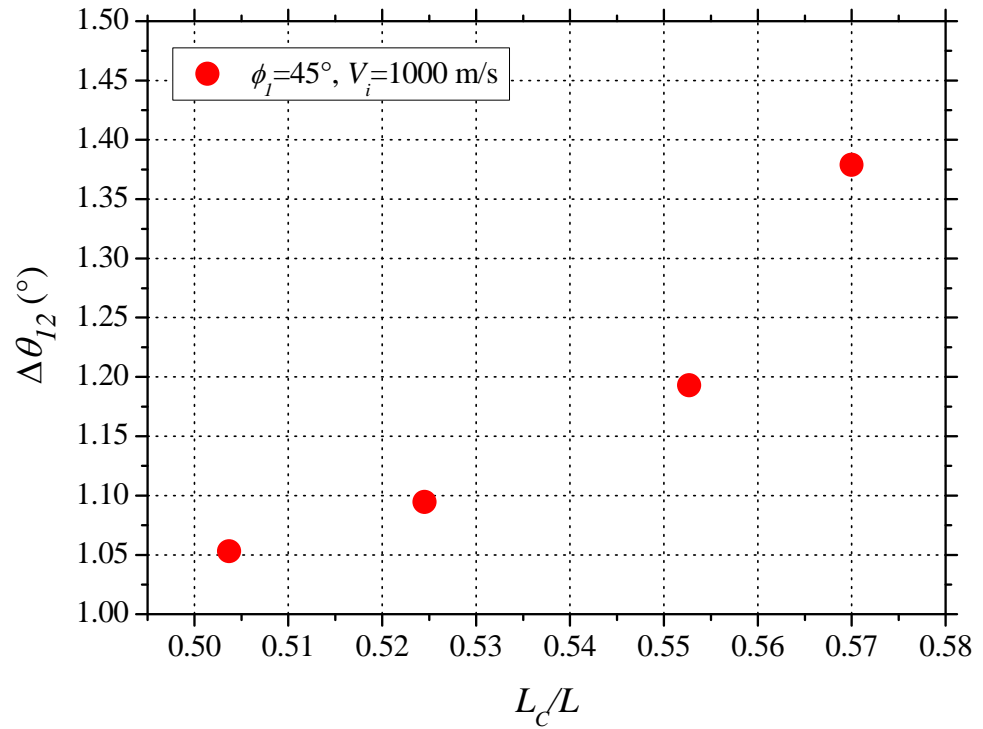

Fig.9 Total direction change $\left(\Delta \theta_{12}\right)$ against $L_{C} / \mathrm{L}$ for an angle of obliquity $\phi_{1}=45^{\circ}$ and impact velocity $V_{i}=1000 \mathrm{~m} / \mathrm{s}$.

The influence of yaw angle on the trajectory of oblique impact is also investigated. The yaw angle $\phi_{2}$ is defined as the difference between the velocity vector angle $\alpha$ and the projectile angle of obliquity $\phi_{1}$ (Fig.10) [Cagliostro et al.(1990)]. Yaw angles between -4 and $4^{\circ}$ are investigated which represent real cases reported experimentally [Goldsmith(1999)]. Two angles of obliquity $30^{\circ}$ and $60^{\circ}$ and projectiles \#2 and \#4 are used. Two different trajectories are identified which are depicted in Fig.11. For all negative yaw angles, when the $V_{c r}$ is reached, the projectile nose is pointing in the positive $x$-direction $(x=+)$ as it is at the beginning of the simulation. However, for certain positive yaw angles, the projectile is eventually pointing in the opposite direction ( $x=-$ ) (Fig.10). This change in trajectory direction has been observed in penetration of blunt projectiles into geological media [(Simonov and Osipenko(2004)]. Figure 12 shows the dependency of $V_{c r}$ on $\phi_{1}, \phi_{2}$ and $L_{c} / L$. It can be seen that $V_{c r}$ decreases with the increase of $L_{c} / L, \phi_{1}$ or $\phi_{2}$ when other parameters are fixed. It can also be seen in Fig.12 that the occurrence of change of trajectory from $x=+$ to $x=-$ is more likely to happen for small angles of obliquity $\left(30^{\circ}\right)$, large values of $L_{c} / L(0.57)$ and large positive angles of $\phi_{2}\left(>1^{\circ}\right)$ for the projectile geometry studied.

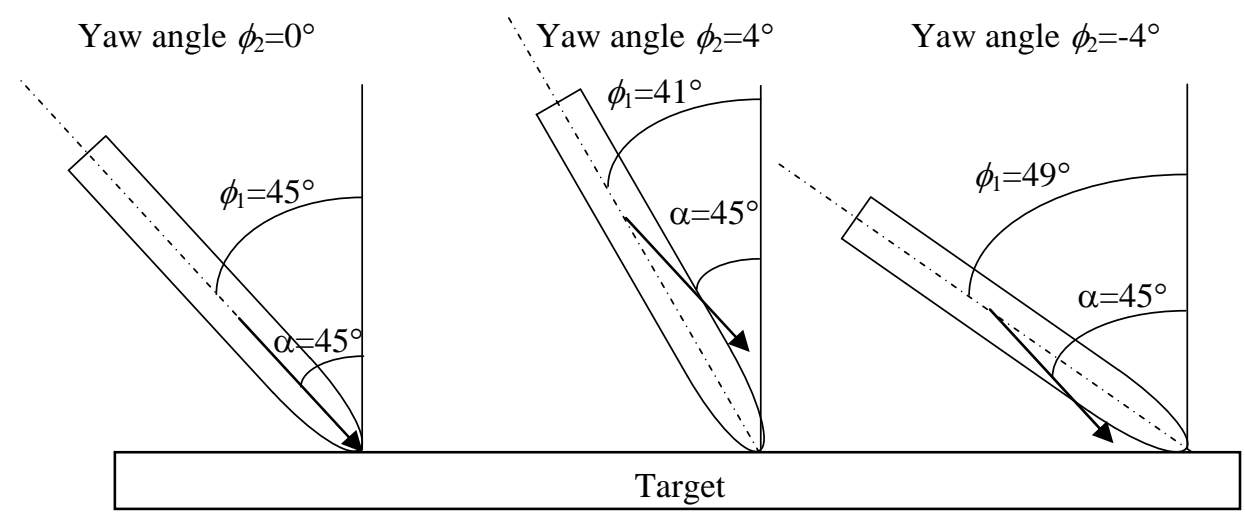

Fig.10 Definition of yaw angle 


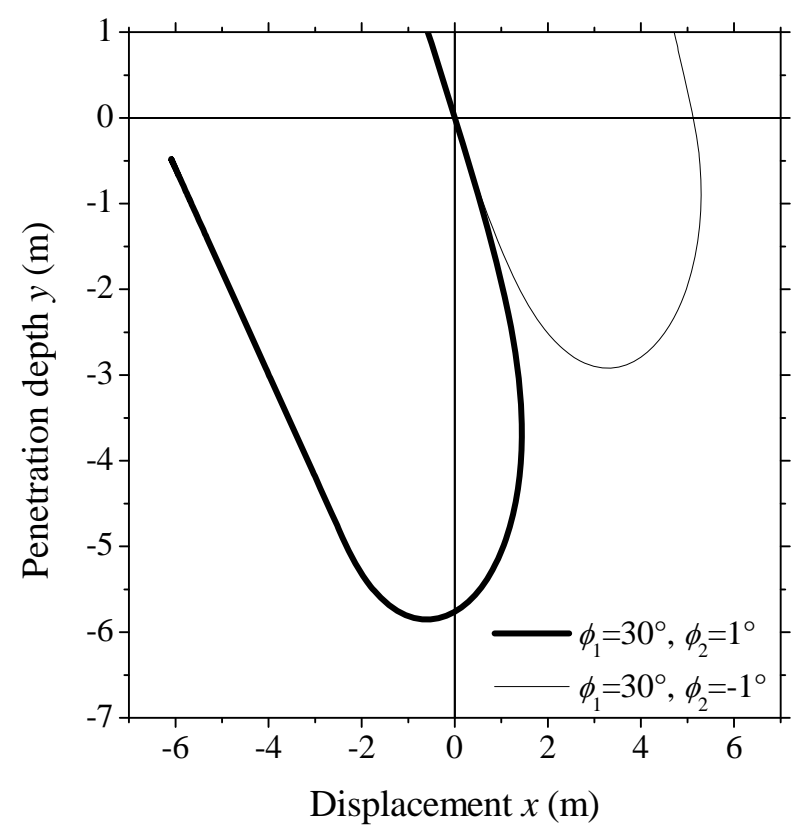

Fig.11 Trajectories of projectile $\# 4$ with $V_{i}=1000 \mathrm{~m} / \mathrm{s}$ and $\phi_{1}=30^{\circ}$ for yaw angles $\phi_{2}$ of -1 and $1^{\circ}$.

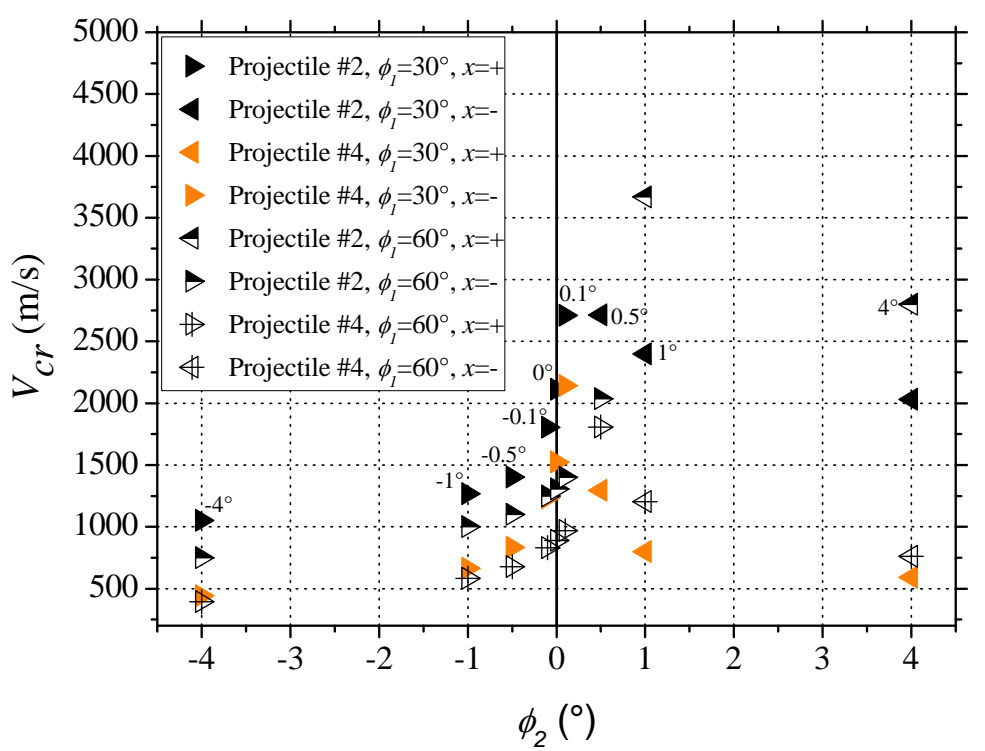

Fig.12 Critical reverse velocity against yaw angle

\subsection{Trajectory instability}

The effect of disturbances (small yaw angle and angular velocity) and large yaw angles on the trajectory instability of normal impact are investigated. Figure 13 shows the trajectories of projectile \#4 with a yaw angle of $0.1^{\circ}$ and various initial normal impact velocities. It can be seen that for impact velocities of 280 and $1000 \mathrm{~m} / \mathrm{s}$ the trajectory deviation $\varphi$ from the normal direction is $0.16^{\circ}$ and $0.24^{\circ}$, respectively, where $\varphi=90^{\circ}+\tan ^{-1}(\mathrm{~d} x / \mathrm{d} y)$ (Figs.13, 14). This indicates that the initial disturbance does not really affect the projectile trajectory until the impact velocity $V_{i}$ is very close to a critical impact velocity $V_{c i}$, from which the projectile largely deviates from the normal trajectory and may move toward to the target surface. $V_{c i}$, as the critical impact velocity for the 
occurrence of instability, is different from $V_{c r}$ which represents the critical impact velocity when the projectile starts to turn back toward the surface in non-axisymmetric penetration problems (Section 4.2). Figure 15 shows the dependency of the critical impact velocity $V_{c i}$ on $L_{c} / L$ for various yaw angles. It can be seen that for a given yaw angle, $V_{c i}$ decreases with the increase of $L_{c} / L$ or $\phi_{2}$ when other parameters are fixed. This dependency of the instability trajectory on the yaw angle for normal impact has been observed experimentally for cone-shaped projectiles and soil-type medium [Osipenko(2009)]. $L_{c} / L$ is again a key factor to control the occurrence of projectile instability.

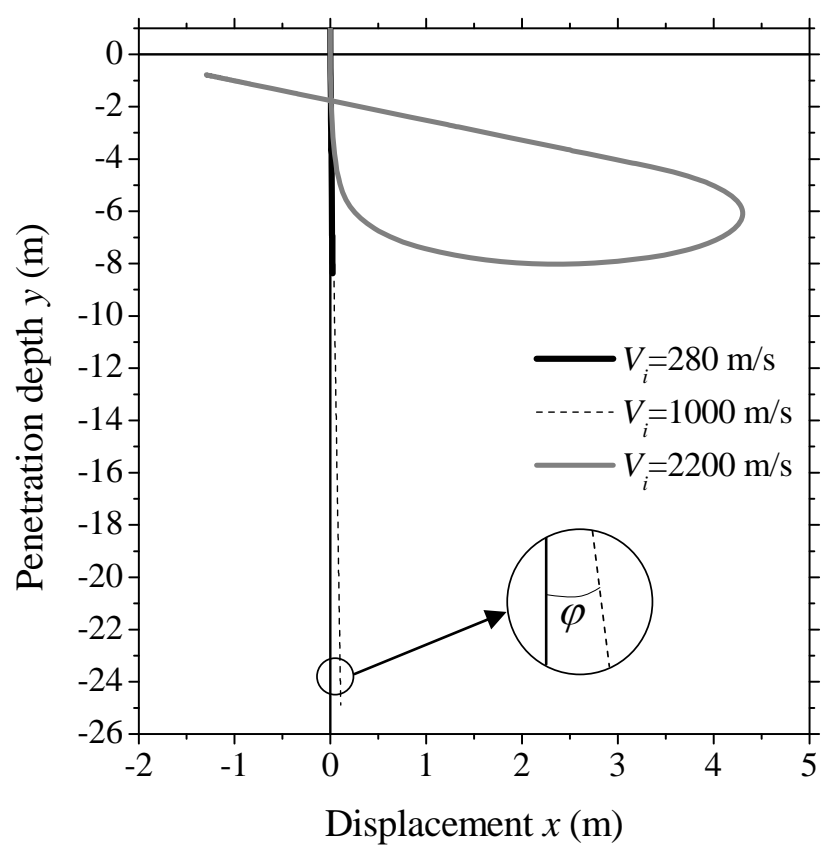

Fig.13 Trajectories of projectile \#4 with a yaw angle of $0.1^{\circ}$ and various initial normal velocities
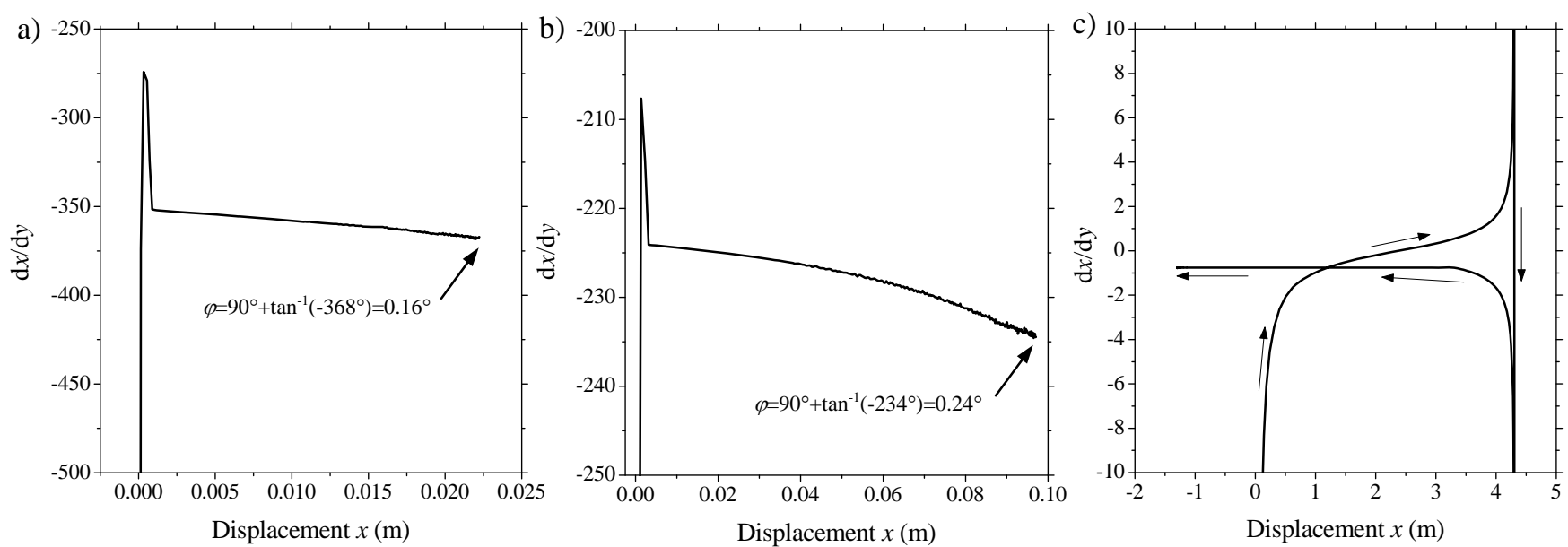

Fig.14 First derivative $\mathrm{d} x / \mathrm{d} y$ of the trajectories from Fig.13:

(a) $V_{i}=280 \mathrm{~m} / \mathrm{s}$, (b) $V_{i}=1000 \mathrm{~m} / \mathrm{s}$, (c) $V_{i}=2200 \mathrm{~m} / \mathrm{s}$ 


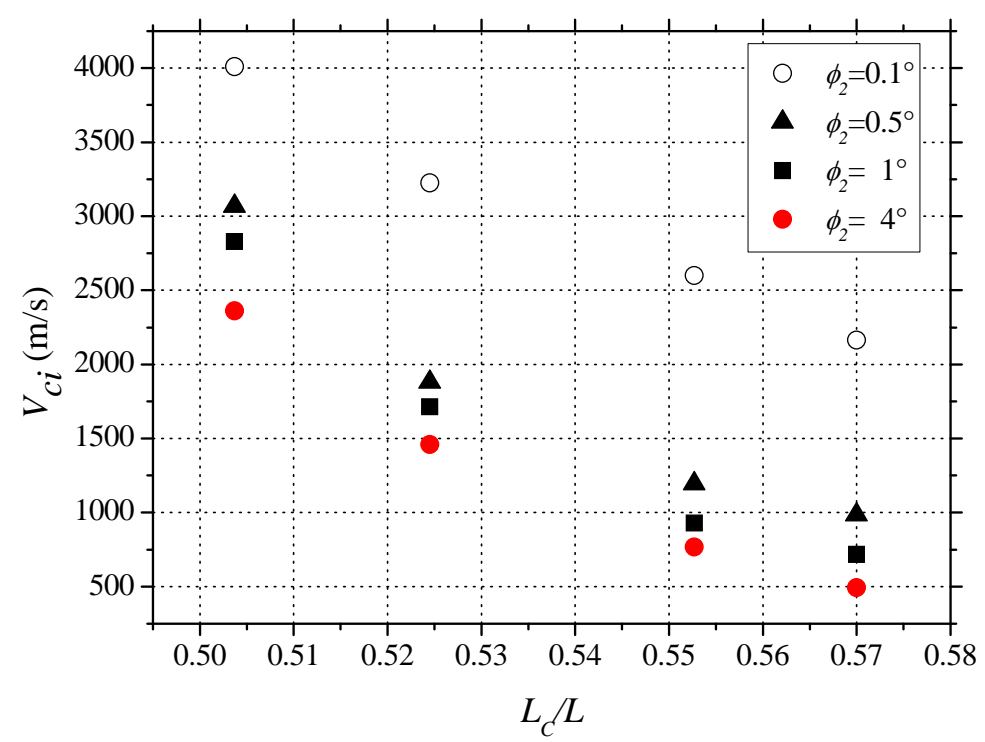

Fig.15 Critical impact velocity against $L_{c} / L$ for various yaw angles

Figure 16 shows the trajectories of projectile \#2 with an initial impact velocity of $1000 \mathrm{~m} / \mathrm{s}$ and various initial angular velocities $\omega$ about the centre of mass. It can be seen that for $\omega=5 \mathrm{rad} / \mathrm{s}$, the deviation of trajectory from the normal direction is negligible $\left(\alpha=0.14^{\circ}\right)$ [Fig.17(a)]. The $\mathrm{d} x / \mathrm{d} y$ of this trajectory [Fig.17(a)] is very similar to the $\mathrm{d} x / \mathrm{d} y$ of the trajectories in Fig.14(a-b). For $\omega=20$ $\mathrm{rad} / \mathrm{s}$, the deviation of the trajectory is considerably large $\left(\alpha=2.86^{\circ}\right)$ [Fig.17(b)]. The $\mathrm{d} x / \mathrm{d} y$ shows that this trajectory goes from curved to linear. For $\omega=25 \mathrm{rad} / \mathrm{s}$, the projectile moves back to the surface in a curved path which becomes linear after a long penetration distance. A critical angular velocity $\omega_{c}$ can be defined as the angular velocity in which the projectile turns back to the surface for a given initial impact velocity $V_{i}$. Figure 18 shows the dependency of $\omega_{c}$ on the $V_{i}$ for various $L_{c} / L$. It is observed that $\omega_{c}$ decreases with the increase of $V_{i}$ or $L_{C} / L$ when other parameters are fixed.

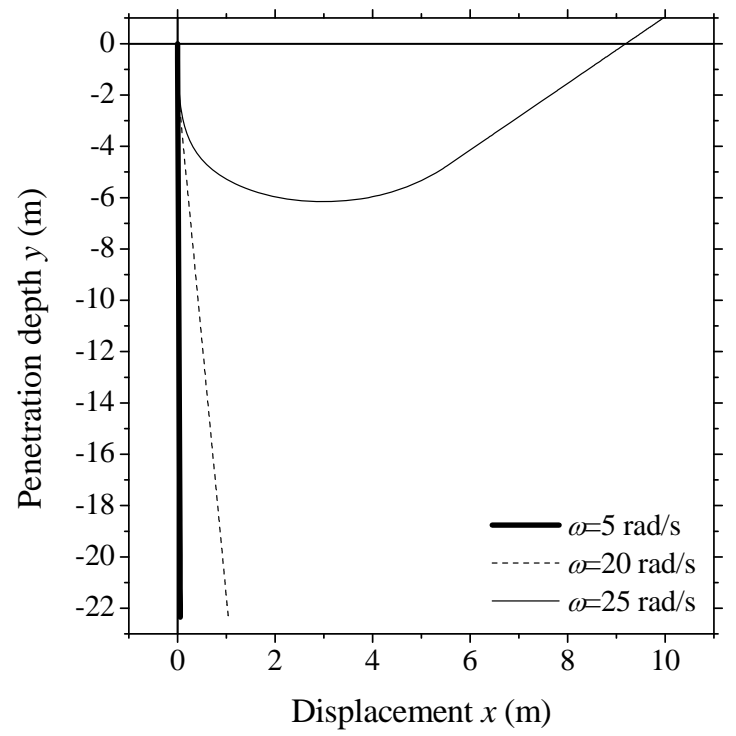

Fig.16 Trajectories of projectile \#2 with an initial impact velocity of $1000 \mathrm{~m} / \mathrm{s}$ and various angular velocities 

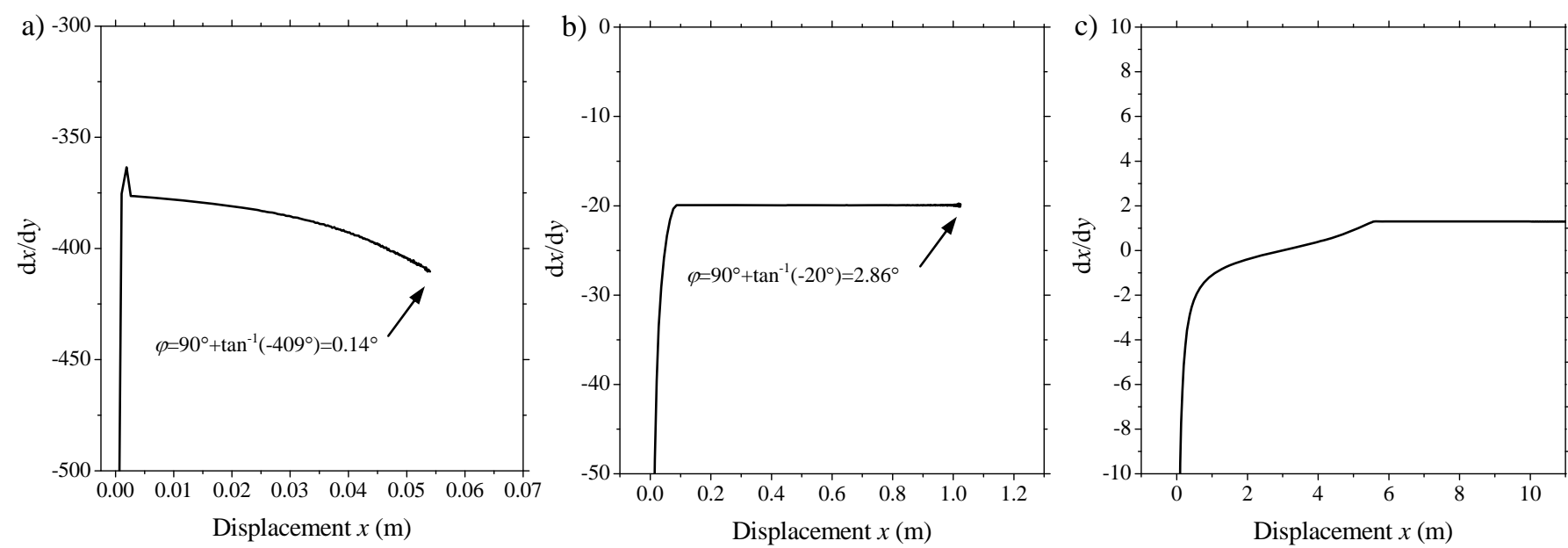

Fig.17 First derivative dx/dy of the trajectories from Fig.16: (a) $\omega=5 \mathrm{rad} / \mathrm{s}$, (b) $\omega=20 \mathrm{rad} / \mathrm{s}$, (c) $\omega=25 \mathrm{rad} / \mathrm{s}$

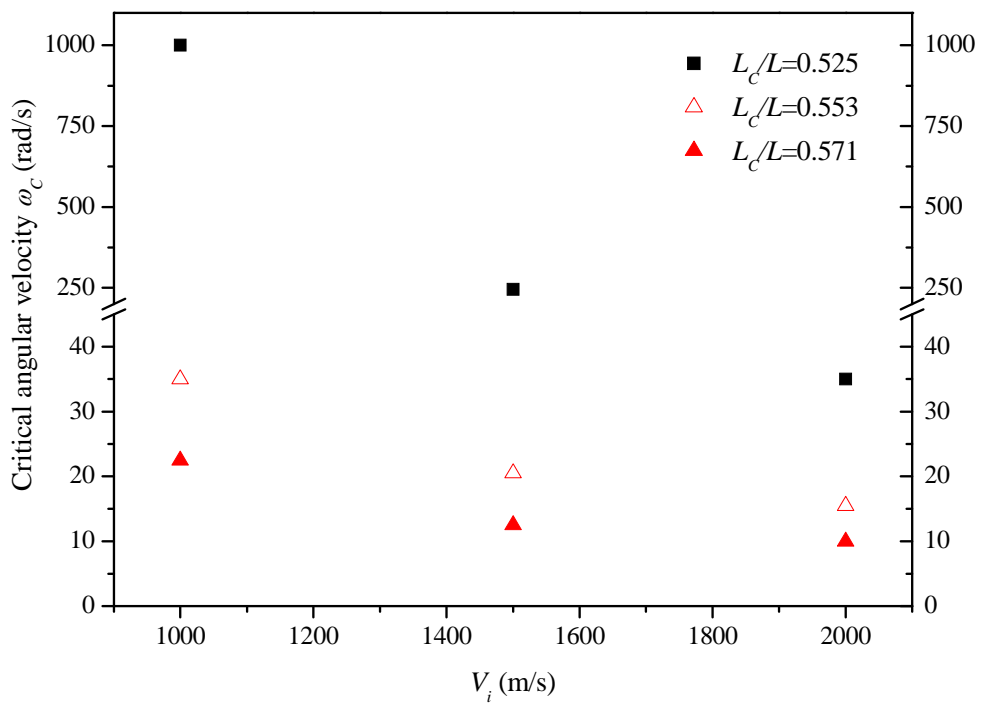

Fig.18 Angular critical velocity against initial impact velocity for various $L_{c} L$

\section{Conclusive remarks}

A general framework of rigid projectile penetration is presented and implemented through ABAQUS to study the projectile trajectory in soil medium as an example. It shows that the curvilinear motion of the projectile may occur due to the occurrence of instability during penetration or the initial unbalanced lateral force as a result of large oblique and/or yaw angles. The relative location of mass centre of the projectile represented by $L_{c} / L$ has strong influence on the control of the rotation of the projectile, and thus, the projectile stability and the change of trajectory direction.

Free surface and wake separation and reattachment are not considered in this paper. They may become important under certain circumstances [Bernard and Creighton(1979)], which, however, have not yet been clarified. Further investigations on these issues are necessary. 
Although numerical modelling based on discrete element method has been widely applied in penetration analysis, it requires extensive material parameters at high strain-rates, large strain, high pressure and high temperature. Enormous resources are needed for the determination of these parameters and considerable uncertainties are unavoidable. The use of a simple analytical model presents a viable alternative to these analyses, at a fraction of the experimental and computational expenses.

Acknowledgements: First author acknowledges the support from State Key Laboratory of Explosion Science and Technology, Beijing Institute of Technology.

\section{References}

Abaqus(2004), Analysis user's manual, Version 6.5.

Adley MD, Berger RP, Cargile JD, White HG, Greighton DC(1997), Three dimensional projectile penetration into curvilinear geological/structural target, User's guide for PENCRV3D, US Army Waterways Experiment Station, Instruction Report SL-97-1, Vicksbury, M.S., January, 1997.

Ahmad Zaidi AM, Li QM(2009), Investigation on penetration resistance of foamed concrete, Proceeding of the Institute of Civil Engineering, Structures and Buildings, 162, 77-85.

Bernard RS, Creighton DC(1978), Non-normal impact and penetration: analysis for hard targets and small angles of attack. Technical report S-78-14. U.S. Army Engineer Waterways Experiment Station Soils and Pavements Laboratory.

Bernard RS, Creighton DC(1979), Projectile penetration in soil and rock: analysis for non-normal impact. Technical report SL-79-15. U.S. Army Engineer Waterways Experiment Station Structures Laboratory.

Bless SJ, Sataphaty S, Normandia MJ(1999), Transverse loads on a yawed projectile, Int. J Engng. Sci., 23, 77-86.

Byers RK, Yarrington P, Chabai AJ(1978), Dynamic penetration of soil media by slender projectiles, Int. J Engng. Sci., 16, 835-844.

Cagliostro DJ, Mandell DA, Schwalbe LA, Adams TF, Chapyak EJ(1990), MESA 3-D calculations of armor penetration by projectiles with combined obliquity and yaw, Int. J. of Impact Engng., 10, 81-92.

Chen XW, Li QM(2002), Deep penetration of a non-deformable projectile with different geometrical characteristics, Int. J. of Impact Engng., 27, 619-637.

Chen XW, Li QM(2004), Transition from nondeformable projectile penetration to semihydrodynamic penetration, ASCE J. of Engng. Mechanics, 130(1), 123-127.

Danielson KT and Adley MD(2000), A meshless treatment of three-dimensional penetrator targets for parallel computation, Comptational Mechanics, 25, 267-273.

Dorogoy A, Rittel D, Brill A(2010), A study of inclined impact in polymethylmethacrylate plates, Int. J. of Impact Engng., 37, 285-294.

Forrestal MJ, Luk VK(1992), Penetration into soil targets, Int. J Impact Engng., 12, 427-44.

Forrestal MJ, Altman BS, Cargile JD, Hanchak SJ(1994), An empirical equation for penetration depth of ogive-nose projectiles into concrete targets, Int J Impact Engng, 15(4), 395-405.

Forrestal MJ, Piekutowski AJ(2000), Penetration experiments with 6061-T6511 aluminum targets and spherical-nose steel projectiles at striking velocities between 0.5 and $3.0 \mathrm{~km} / \mathrm{s}$, Int J Impact Engng, 24, 57-67.

Goldsmith W(1999), Non-ideal impact projectile on targets, Int J Impact Engng, 22, 95-395.

Heuze FE(1990), An overview of projectile penetration into geological materials, with emphasis on 
rocks, Int. J. of Rock Mechanics and Mining Sciences and Geomechanics Abstracts, Vol.27(1), 114.

Li QM, Chen XW(2003), Dimensionless formulae for penetration depth of concrete target impacted by a non-deformable projectile, Int. J. of Impact Engng., 28, 93-116.

Li QM(2004), A framework of penetration mechanics for hard projectile, Applied Advances in Mechanics. Editor YS Yong, Science Press, Beijing, 261-270.

Li QM, Maharaj RN, Reid SR (2005), Penetration resistance of aluminium foam, Int. J. Vehicle Design, 37(2/3), 175-184.

Lindberg HE, Florence AL(1987), Dynamic pulse buckling, Martinus Nijhoff Publishers, Dordrecht. Liu Y, Ma A, Huang F(2009), Numerical simulations of oblique-angle penetration by deformable projectiles into concrete targets, Int J Impact Engng, 36, 438-446.

Osipenko KY(2009), Penetration of a body of revolution into an elastoplastic medium. Mechanics of Solids, 44(2), 311-321.

Piekutowski AJ, Forrestal MJ, Poormon KL, Warren TL(1999), Penetration of 6061-T6511 aluminum targets by ogive-nose steel projectiles with striking velocities between 0.5 and $3.0 \mathrm{~km} / \mathrm{s}$, Int J Impact Engng, 23, 723-734.

Rosenberg Z, Surujon Z, Yeshurun Y, Ashuach Y, Dekel E(2005), Ricochet of 0.3” AP projectile from inclined polymeric plates, Int J Impact Engng, 31, 221-233.

Simonov IV, Osipenko KY(2004), Stability, paths and dynamic bending of a blunt body of revolution penetrating into an elastoplastic medium, J. Appl. Mech. Tech. Phys., 45(3), 428-439.

Warren TL, Hanchack SJ, Poormon KL(2004), Penetration of limestone targets by ogive-nosed VAR 4340 steel projectiles at oblique angles: experiments and simulations, Int J Impact Engng, 30, 1307-1331. 